\begin{tabular}{|c|c|}
\hline Title & Double threshold behavior in a resonance controlled $\mathrm{ZnO}$ random laser \\
\hline Author(s) & Niyuki, Ryo; Fujiwara, Hideki; Nakamura, Toshihiro; Ishikawa, Y oshie; Koshizaki, Naoto; Tsuji, T akeshi; Sasaki, Keiji \\
\hline Citation & $\begin{array}{l}\text { A pl photonics, 2(3), } 36101 \\
\text { https://doi.org/10.1063/1.4974334 }\end{array}$ \\
\hline Issue Date & 2017-03 \\
\hline Doc URL & http:/hdl.handle.net/2115/65347 \\
\hline Rights & Copyright 2017 A uthor(s). This article is distributed under a Creative Commons A ttribution (CC BY) License. \\
\hline Rights(URL) & http://creativecommons.org/icenses/by/4.0/ \\
\hline Type & article \\
\hline File Information & 1.4974334.pdf \\
\hline
\end{tabular}

Instructions for use 


\section{Double threshold behavior in a resonance-controlled $\mathrm{ZnO}$ random laser}

Ryo Niyuki, Hideki Fujiwara, Toshihiro Nakamura, Yoshie Ishikawa, Naoto Koshizaki, Takeshi Tsuji, and Keiji Sasaki

Citation: APL Photonics 2, 036101 (2017); doi: 10.1063/1.4974334

View online: http://dx.doi.org/10.1063/1.4974334

View Table of Contents: http://aip.scitation.org/toc/app/2/3

Published by the American Institute of Physics

\section{Articles you may be interested in}

Efficient continuous-wave nonlinear frequency conversion in high-Q gallium nitride photonic crystal cavities on silicon

APL Photonics 2, 031301031301 (2017); 10.1063/1.4974311

Why I am optimistic about the silicon-photonic route to quantum computing

APL Photonics 2, 030901030901 (2017); 10.1063/1.4976737

Compact and high-resolution optical orbital angular momentum sorter

APL Photonics 2, 031302031302 (2017); 10.1063/1.4974824

Plasmonic silicon Schottky photodetectors: The physics behind graphene enhanced internal photoemission APL Photonics 2, 026103026103 (2017); 10.1063/1.4973537

Large static tuning of narrow-beam terahertz plasmonic lasers operating at $78 \mathrm{~K}$

APL Photonics 2, 026101026101 (2016); 10.1063/1.4972127

Nanoboomerang-based inverse metasurfaces_-A promising path towards ultrathin photonic devices for transmission operation

APL Photonics 2, 036102036102 (2017); 10.1063/1.4974343

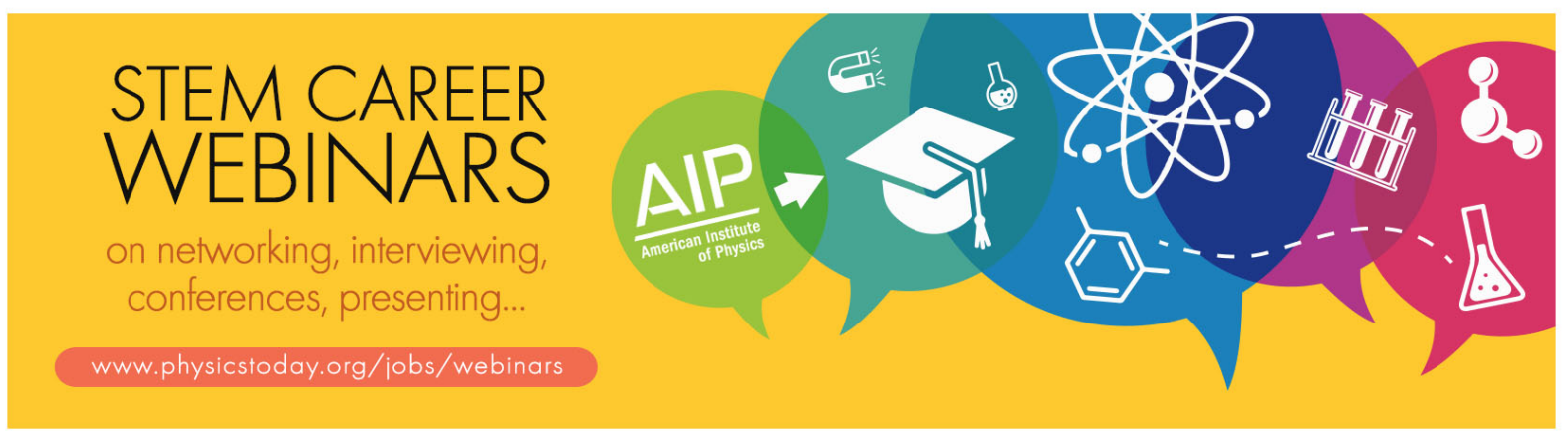




\title{
Double threshold behavior in a resonance-controlled ZnO random laser
}

\author{
Ryo Niyuki, ${ }^{1}$ Hideki Fujiwara, ${ }^{1, a, b}$ Toshihiro Nakamura, ${ }^{2, b, c}$ Yoshie Ishikawa, ${ }^{3}$ \\ Naoto Koshizaki, ${ }^{4}$ Takeshi Tsuji, ${ }^{5}$ and Keiji Sasaki ${ }^{1}$ \\ ${ }^{1}$ Research Institute for Electronic Science, Hokkaido University, Sapporo, Hokkaido 001-0020, \\ Japan \\ ${ }^{2}$ Division of Electronics and Informatics, Faculty of Science and Technology, Gunma University, \\ Kiryu, Gunma 376-8515, Japan \\ ${ }^{3}$ National Institute of Advanced Industrial Science and Technology, Tsukuba, \\ Ibaraki 305-8565, Japan \\ ${ }^{4}$ Division of Quantum Science and Engineering, Faculty of Engineering, Hokkaido University, \\ Sapporo, Hokkaido 060-8628, Japan \\ ${ }^{5}$ Interdisciplinary Graduate School of Science and Engineering, Shimane University, Matsue, \\ Shimane 690-8504, Japan
}

(Received 28 September 2016; accepted 6 January 2017; published online 25 January 2017)

\begin{abstract}
We observed unusual lasing characteristics, such as double thresholds and blue-shift of lasing peak, in a resonance-controlled $\mathrm{ZnO}$ random laser. From the analysis of lasing threshold carrier density, we found that the lasing at 1 st and 2 nd thresholds possibly arises from different mechanisms; the lasing at 1st threshold involves exciton recombination, whereas the lasing at 2nd threshold is caused by electron-hole plasma recombination, which is the typical origin of conventional random lasers. These phenomena are very similar to the transition from polariton lasing to photon lasing observed in a well-defined cavity laser. (C) 2017 Author(s). All article content, except where otherwise noted, is licensed under a Creative Commons Attribution (CC BY) license (http://creativecommons.org/licenses/by/4.0/). [http://dx.doi.org/10.1063/1.4974334]
\end{abstract}

Zinc oxide $(\mathrm{ZnO})$ has been one of the attractive materials because of its excellent high emission efficiency, high electrical conductivity, high binding energy, transparency in the visible region, and wide emission range, which has been utilized for various applications, such as light emitting devices, solar cells, transistors, and photocatalysts. So far, as gain materials, many studies on UV $\mathrm{ZnO}$ lasers have also been reported in various micro-/nano-cavity structures, such as random structures, ${ }^{1-13}$ Fabry-Perot cavities, ${ }^{14-17}$ whispering-gallery-mode resonators. ${ }^{18-21}$ Although, in most of these $\mathrm{ZnO}$ lasers, the gain from electron-hole plasma (EHP) recombination under high excitation intensity condition has typically been reported as the origin of the stimulated emission (photon lasing), ${ }^{12,13}$ the lasing originated by excitonic recombination, so-called polariton/exciton lasers, has also been demonstrated in well-designed cavity structures at room temperature. ${ }^{21-23}$

Random lasers (RLs) based on photon localization due to multiple light scattering have been reported in various materials. In particular, because $\mathrm{ZnO}$ has the above-mentioned advantages and high gain, there are numerous studies on RLs so far. ${ }^{1-13}$ In typical random structures, because localized modes are spontaneously formed and its modal control is difficult due to the multiple light scattering, the lasing peak wavelengths depend mainly on the property of gain materials and their thresholds are determined by the mean scattering property of the structure. Furthermore, the features of the RL (e.g., multimode lasing and high thresholds) make it difficult to realize strong interactions between cavity modes and gain materials and observe cavity-related phenomena, ${ }^{21-24}$ like observed in well-designed resonators, as mentioned above.

\footnotetext{
afuji@es.hokudai.ac.jp

$\mathrm{b}_{\mathrm{H}}$. Fujiwara and T. Nakamura contributed equally to this work.

cnakamura@el.gunma-u.ac.jp
} 
On the contrary, we have proposed a new RL device consisting of size-monodispersive scatterers and intentionally introducing scatterer free sites, which exhibit unique properties realizing quasi-single-mode, low threshold, and lasing wavelength control, due to the resonance of individual scatterers. ${ }^{25-27}$ Note that, hereafter, the intentionally introduced scatterer free site and the new RL devices are referred to as "defect site" and "resonance-controlled RLs." Because our proposed RLs can realize low-threshold and single-mode random lasing, which are quite distinct from conventional RLs, the photophysical phenomena related to $\mathrm{ZnO}$ excitons that are difficult to observe in conventional RLs could also be induced even in random structures, due to the improvement of photon localization by the use of resonant scatterers. In fact, in our previous study, ${ }^{13}$ measuring the temperature dependence of the resonance-controlled RL properties, we found that the lasing origin was quite different from conventional RLs and suggested the possibility that the resonance-controlled RLs were related to exciton lasing, like well-designed microcavities, ${ }^{21-23}$ whereas the origin of conventional ZnO RLs was caused by EHP recombination. ${ }^{13,26}$

In this study, we observe the peculiar features (double threshold behavior, blue peak shift, and peak width change) of single-mode lasing in the resonance-controlled ZnO RL. These features are similar to the polariton lasers and have been never before reported in conventional RLs. From the carrier density analysis, we find that the lasing at 1 st and 2 nd thresholds is dominated by different mechanisms and suggest the possibility that the lasing at 1st threshold involves excitonic recombination because the carrier density is almost corresponding to or less than the Mott transition density, whereas the 2nd threshold lasing is caused by usual EHP recombination that is the typical origin of conventional RLs.

The experimental setup and sample preparation are the same as that used in our previous study. ${ }^{25-27}$ We used quasi-mono-dispersive $\mathrm{ZnO}$ nanoparticles (NPs) as scatterers and gain materials, which were fabricated from commercially available $\mathrm{ZnO}$ NPs with a mean diameter of $100 \mathrm{~nm}$ by using a laser-induced melting method. ${ }^{28}$ After the fabrication, spherical ZnO NPs with a diameter of $\sim 212 \mathrm{~nm}$ were obtained, which were almost mono-dispersive and spherical, in contrast to the commercially available poly-dispersive ZnO NPs with irregular shapes. Dispersing these ZnO NPs and green fluorescent polystyrene particles with a diameter of $900 \mathrm{~nm}$ as point defects in water, the solution was casted on a cover glass and dried. Similarly, as reference, poly-dispersive ZnO NP films were also prepared. Then, the sample fixed on a microscope stage was excited by a Q-switched pulsed laser (wavelength $355 \mathrm{~nm}$, repetition rate $1 \mathrm{kHz}$, pulse duration $300 \mathrm{ps}$, spot size $\sim 65 \mu \mathrm{m}$ ) and measured the emission spectra from around a defect site. The location of defect site was confirmed from a green fluorescence image of polystyrene particles. Emission from the sample passing through a pinhole (about $1 \mu \mathrm{m}$ on the sample plane) was detected using a spectroscope equipped with a cooled CCD camera. We repeatedly measured the excitation dependence of emission spectra at different defect sites.

Figures 1(a) and 1(c) show a SEM image, an emission image, and emission spectra of conventional random lasing in a poly-dispersive $\mathrm{ZnO}$ NP film. The emission image shows almost uniform distribution in the excitation spot, regardless of the defect sites. In the emission spectra, multiple sharp peaks superposed on a collapsed broad emission spectrum along with the red-shift of these peaks are observed around $387 \mathrm{~nm}$ (conventional RLs). In our previous study, ${ }^{13,26}$ we revealed that the conventional RLs were caused by the gain due to EHP recombination, and the lasing wavelength was determined by this gain property of $\mathrm{ZnO}$, not by the optical property of random structure. Thus, the red-shift of the lasing peaks reflects the excitation intensity dependence of the $\mathrm{ZnO}$ gain peak.

In the mono-dispersive spherical ZnO NP film (Figs. 1(b) and 1(d)), we observe random lasing with single lasing peak induced in a narrowed area as shown in the emission image (resonancecontrolled RLs). ${ }^{25}$ The single lasing peak appears around the wavelength of $380 \mathrm{~nm}$. This peak position is clearly different from the maximum of the spontaneous emission spectrum. With increasing the excitation intensity, the lasing peak wavelength shifts toward a shorter wavelength side (inset of Fig. 1(d)). Furthermore, the threshold of the resonance-controlled RL (several $\mathrm{MW} / \mathrm{cm}^{2}$ ) is one order of magnitude lower than that of the conventional RL (several tens $\mathrm{MW} / \mathrm{cm}^{2}$ ). Thus, we can understand that the lasing characteristics of the resonance-controlled RL are determined by the resonant properties of proposed random structure (i.e., spherical resonant scatterers used), not only the $\mathrm{ZnO}$ gain properties. ${ }^{25}$ 

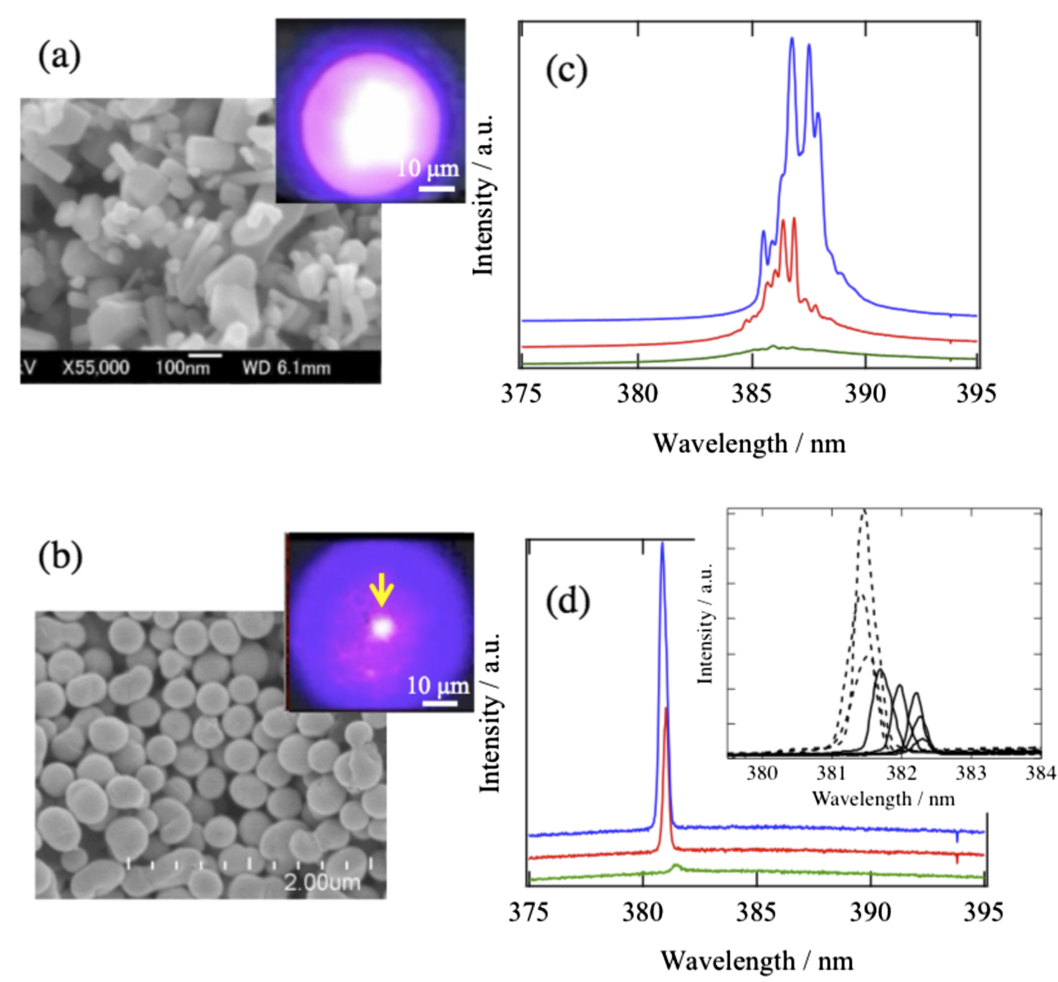

FIG. 1. SEM images of (a) poly-dispersive and (b) mono-dispersive ZnO NPs. The insets in (a) and (b) show emission images of UV random lasing from each $\mathrm{ZnO}$ NP film. Arrow indicates the position of the defect site. Lasing emission spectra with different excitation intensities of (c) conventional and (d) resonance-controlled RLs are shown. The inset in (d) shows the enlarged view of the emission spectra around $382 \mathrm{~nm}$.

In the resonance-controlled RLs, we occasionally observed peculiar lasing behaviors. Figure 2 shows the excitation intensity dependence of the intensity (upper part), wavelength (lower part, right axis), and width (lower part, left axis) of the lasing peak in Fig. 1(d). From the results, we found double threshold behavior, 1st threshold at $\sim 5 \mathrm{MW} / \mathrm{cm}^{2}$ and 2 nd threshold at $\sim 20 \mathrm{MW} / \mathrm{cm}^{2}$. The 1st threshold value is about 10 times lower than conventional RLs, and the 2nd threshold value is slightly lower than those of conventional RLs. Above the 1st threshold, the single lasing peak continuously blue-shifts with increasing excitation intensity between the 1st and 2nd thresholds and starts to broaden at $10 \mathrm{MW} / \mathrm{cm}^{2}$ (also see solid lines in the inset of Fig. 1(d)). On the contrary, above the 2 nd threshold, lasing spectra remain single peak and the blue-shift stopped. We should note that the spontaneous emission peak shows a red-shift with increasing excitation intensity due to a band-gap renormalization effect as similar to conventional ZnO RLs. ${ }^{13}$ These peculiar characteristics (double threshold behavior, blue-shift of lasing peak) in the resonance-controlled RLs indicate that the underlying physical origins of its lasing oscillation are distinct from the conventional RLs.

To confirm the reproducibility, we repeatedly measured the lasing properties at different defect sites. Figure 3 shows the scatter plots of the lasing peak wavelengths and their thresholds. Circles indicate the thresholds and lasing wavelengths of the resonance-controlled RLs; especially the data exhibiting double threshold behaviors were indicated as open circles. To compare the lasing properties, we also plotted those of conventional RLs as diamonds, in which the center wavelengths of the multi-mode lasing were plotted. From the results, we can confirm the similar tendency as shown in Figs. 1 and 2, double threshold, blue-shifted lasing wavelength, and low-threshold compared to the conventional RLs. Furthermore, we also plotted the data measured at defect-free sites in the resonance-controlled RLs (solid squares in Fig. 3) and found that it exhibited multi-mode lasing with lasing peak red-shift and higher thresholds, similar to the conventional RLs. These results indicate that no localized modes resulting in the strong photon confinement were induced at the defect-free sites as mentioned in our previous studies. ${ }^{25-27,29}$ We note that a resonance-controlled 


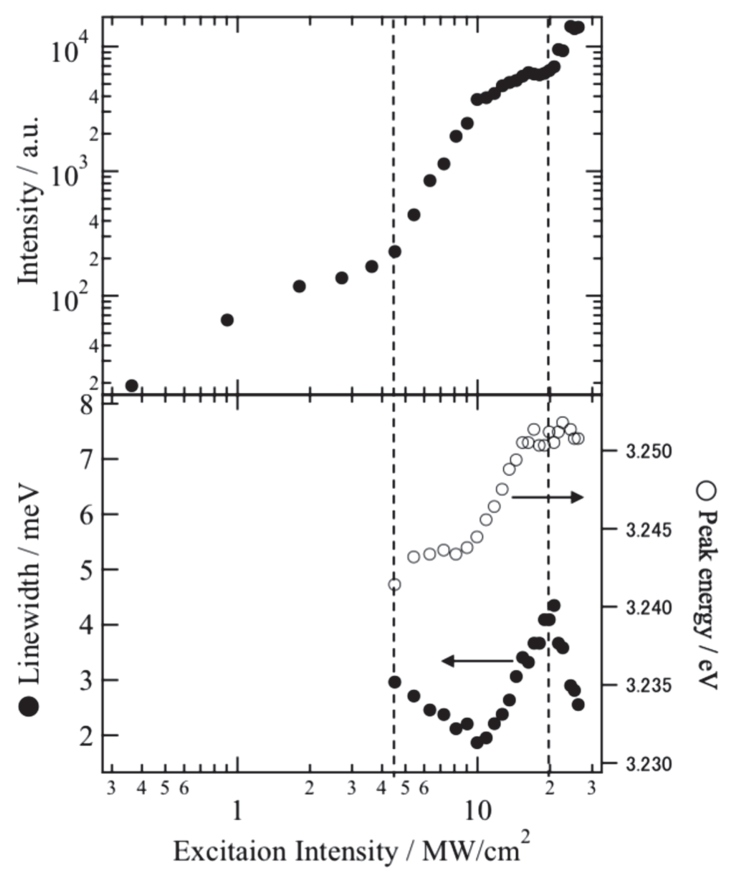

FIG. 2. Excitation intensity dependence of intensity, energy, and width of the lasing peak for the resonance-controlled RL (Fig. 1(d)). Dashed lines indicate the 1st and 2nd thresholds.

random structure (i.e., a defect polystyrene particle and surrounding ZnO NPs) was occasionally damaged by the excitation laser irradiation at several tens $\mathrm{MW} / \mathrm{cm}^{2}$, due to the strong photon confinement, which was close to the 2nd thresholds. This damage may prevent us from observing 2 nd threshold in the resonance-controlled RL, resulting in a small number of the data plots (Fig. 3). We also note that such sample damage is not the origin of the double threshold behavior, since the reproducibility of double threshold behaviors with increasing and decreasing the excitation intensity was confirmed.

To understand such unique double threshold behaviors, according to our previous studies, ${ }^{13,26}$ we calculated the optical gain spectra of bulk $\mathrm{ZnO}$ and lasing threshold carrier density. Details on the calculations can be found in Refs. 13, 14, and 30. The gain spectrum $g(\omega)$ calculated by using its

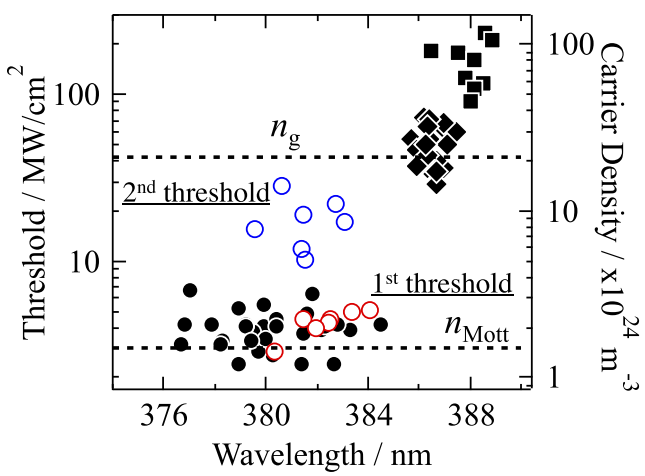

FIG. 3. Scatter plot of lasing peak wavelengths and thresholds. Circles and squares indicate the data at defect and defect-free sites in resonance-controlled RLs, respectively. Especially, open circles show the data exhibiting double threshold behaviors (red: 1st thresholds, blue: 2nd thresholds). Diamonds represent the lasing threshold for conventional RLs. Note that because the center peak wavelengths of conventional multi-mode RLs were plotted, the data have narrower distribution. Lower and upper dotted lines indicate the Mott density $\left(n_{\text {Mott }}\right)$ and the carrier density where the gain overcomes the loss in bulk $\mathrm{ZnO}$ $\left(n_{g}\right)$, respectively. 


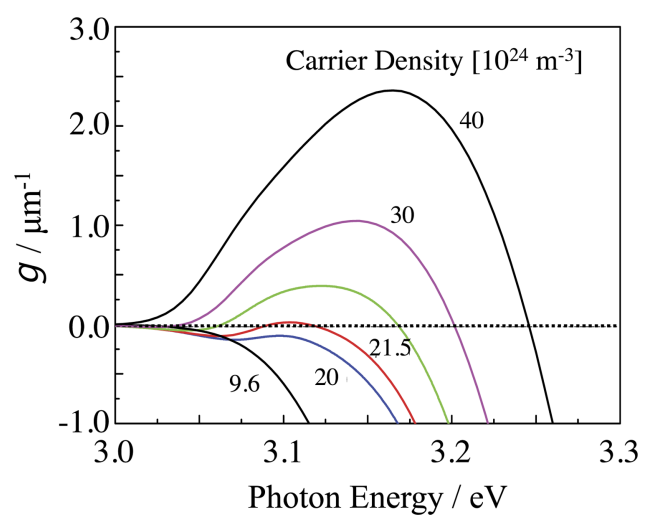

FIG. 4. Calculated gain spectra $g(\omega)$ of bulk $\mathrm{ZnO}$ at various carrier densities $\left(9.6-40 \times 10^{24} \mathrm{~m}^{-3}\right)$. The gain occurs at the carrier density of $2.1 \times 10^{25} \mathrm{~m}^{-3}$.

physical parameters, not using its structural parameters,

$$
g(\omega)=-\frac{2 \omega}{c} \operatorname{Im}[\sqrt{1+\chi(\omega)}],
$$

where $c$ and $\chi(\omega)$ are the speed of light in vacuum and optical susceptibility. $\chi(\omega)$ was obtained by solving the Bethe-Salpeter ladder equation derived from the quantum field theory including the quantum many-body effects of excited carriers. ${ }^{14,30}$ The results of calculated spectra are shown in Fig. 4. From the calculations, the carrier density, where the gain overcomes the losses in the bulk $\mathrm{ZnO}$, was estimated to be $n_{g} \sim 2.1 \times 10^{25} \mathrm{~m}^{-3}$. This value is clearly larger than the Mott density $\left(n_{\text {Mott }} \sim 1.5 \times 10^{24} \mathrm{~m}^{-3}\right),{ }^{14}$ which is one of the criteria for distinguishing excited electronic states between excitonic and EHP states.

The threshold carrier density $n_{\text {th }}\left(\right.$ in $\mathrm{m}^{-3}$ ) was estimated from the excitation intensity and the following differential equation:

$$
\frac{d n(t)}{d t}=\frac{P(t) \eta(T)}{\hbar \omega_{p l} D}-\frac{n(t)}{\tau(T)},
$$

where $n(t), P(t), \hbar \omega_{p l}$, and $D$ denote the time-dependent carrier density, the excited intensity, the photon energy of the excitation light, and the diameter of ZnO NPs, respectively. In the above calculation, we assumed that all incident photons are absorbed by $\mathrm{ZnO}$ particles at the surface of the random structure surface because of the larger photon energy of the excitation light than the bandgap energy of $\mathrm{ZnO}$. In that case, the excited carrier density per unit volume can be given as an incident photon density (i.e., the excited carrier density) per unit area divided by the diameter $(D)$ of $\mathrm{ZnO}$ particles as shown in Eq. (2). The temperature-dependent parameters $\eta(T)$ and $\tau(T)$ represent the intrinsic carrier lifetime and quantum efficiency of $\mathrm{ZnO}$, which were obtained from Refs. 13 and 31. Numerically solving Equation (2) assuming a Gaussian pulse waveform, ${ }^{14,30} n_{\text {th }}$ were obtained as the time-averaged value of $n(t)$ from the experimental $P_{\text {th }}$ values. We note that the $n_{\text {th }}$ values give upper limits because the power losses by the $\mathrm{ZnO}$ random structure were not included in the above procedure and, therefore, the effective carrier densities may become lower inside the structure. From the calculation, the 1st and 2nd threshold carrier densities of Figs. 1 and 2 were estimated as 2.1 $\times 10^{24}$ and $1.0 \times 10^{25} \mathrm{~m}^{-3}$, respectively. The other $n_{\text {th }}$ values are represented by right axis in Fig. 3 .

In Fig. 3, we compared $n_{\text {th }}$ with calculated results ( $n_{g}$ and $n_{\text {Mott }}$, horizontal dashed lines). As shown in the figure, the 1st threshold appeared around $n_{\text {Mott }}$, and the 2 nd threshold was almost one order of magnitude larger than $n_{\text {Mott }}$. Because, as mentioned above, the estimated $n_{\text {th }}$ values are the upper limit, the 1st lasing was likely to be induced below the Mott density. Therefore, it suggests the possibility that the origin of lasing oscillation around 1st threshold in the resonance-controlled RL would be attributed to exciton related emission. ${ }^{26}$ In addition, Chen et al. have reported coherent random lasing action, which is originated from localized exciton gain and strong light scattering, in nanocrystal-embedded $\mathrm{ZnO}$ core-shell nanowires at room temperature. ${ }^{32}$ On the other hand, at 
the 2nd thresholds, the threshold carrier density is much higher than $n_{\text {Mott }}$ and is close to those of the conventional RL. This indicates that the lasing origin above 2nd thresholds is considered to be EHP recombination, as similar to conventional RLs. ${ }^{13,26}$ Even though the same lasing origin as EHP recombination, the 2 nd threshold value is lower than those of conventional RLs. We also should note that the $n_{\text {th }}$ of 2 nd threshold is clearly lower than $n_{g}$. These may be because of the decrease in the effective lasing threshold due to the existence of the localized modes at the defect site in resonance-controlled RLs.

Based on the above analysis of threshold carrier density, we consider the mechanism of the lasing peak shift and width change in Fig. 2. In the case of excitonic lasing at 1st threshold, the blue-shift is attributed to the decrease in the refractive index due to the change in the carrier density with increasing excitation intensity. ${ }^{33,15}$ The similar blue-shift of individual lasing modes originating from the refractive index change was reported in a $\mathrm{ZnO}$ microcavity laser. ${ }^{33}$ Further increasing the excitation intensity, around the 2nd threshold, the peak shift was saturated. This is probably because the red shift caused by bandgap renormalization with increasing the excitation intensity cancels the blue-shift. ${ }^{15}$ The possible mechanism for the increase in the linewidth above $10 \mathrm{MW} / \mathrm{cm}^{2}$ is the refractive index fluctuations of $\mathrm{ZnO}$ and carrier fluctuation with increasing excitation intensity. ${ }^{12,34}$ The spectral narrowing after the 2nd threshold may be due to the transition to photon lasing with population inversion.

On the other hand, we consider another possible mechanism as follows. We confirmed continuous blue-shift of single lasing peak and change in its spectral width with increasing the excitation intensity, without the appearance of other multi-mode lasing peaks from the results in Figs. 1(d) and 2. Furthermore, the analysis of threshold carrier density in Fig. 3 reveals the transition of lasing origin from the exciton related emission to the EHP recombination. These phenomena suggest the possibility that polariton lasing occurs in the resonance-controlled RL that has typically been observed in well-designed microcavity structures..$^{23,24}$ This is because typical polariton lasers show similar features, ${ }^{24}$ such as double threshold behavior (transition from polariton to photon lasers), change in spectral width, and spectral blue-shift. In fact, the tendency of spectral width changes and spectral blue-shift with increasing the excitation intensity in Fig. 1 is almost corresponding to the polariton lasing in well-defined cavities. If polariton lasing is induced in resonance-controlled RLs, the spectral broadening with the excitation intensity above $10 \mathrm{MW} / \mathrm{cm}^{2}$ and blue-shift can be attributed to polariton-polariton (exciton) interaction. In polariton condensate at high density, phase diffusion occurs due to self-interaction, resulting in a loss of coherence. ${ }^{35,36}$ The narrowing after the 2nd threshold also can be explained by the transition to photon lasing with population inversion, as mentioned above. Because not only blue-shift, these peculiar behaviors (double thresholds, continuous blue-shift of single lasing peak, and spectral width change) were observed simultaneously, we consider that the polariton lasing due to the strong coupling between single localized mode and $\mathrm{ZnO}$ excitons induced in the resonance-controlled random structure would be one of the strong candidates for explaining these observed phenomena. However, because it is impossible to measure characteristics of cavity mode dispersion (angle-resolved reflectance or emission spectra) from the resonance-controlled RL due to the multiple light scattering, it is hard to verify the existence of microcavity exciton-polariton. Further investigation is necessary to reveal whether it is a polariton lasing or not, for example, by measuring the dependence of resonance-controlled random lasing on the frequency detuning between the exciton and localized mode by changing the temperature.

There is another possibility for the peculiar behaviors of the present resonance-controlled RL, the transition from the stimulated emission of exciton scattering at the 1st threshold to the population inversion of EHP recombination at the 2nd threshold. Because the transition from exciton-scattering to EHP recombination usually accompanies discontinuous photoluminescence peak red-shifts, ${ }^{37,38} \mathrm{a}$ red-shift of stimulated emission peak is expected to occur. However, in the present case, the lasing peak continuously shifts to a blue-side. Furthermore, distinct double threshold behaviors that we observed have never been reported due to the transition. Thus, such a transition mechanism can be safely excluded as the origin of the peculiar lasing behaviors.

We observed double threshold behaviors and continuous blue-shift of single lasing peaks in our proposed resonance-controlled RLs, which have never before observed in conventional RLs. 
From the results, we found that the 1 st threshold carrier density was comparable to the Mott density $\left(1.5 \times 10^{24} \mathrm{~m}^{-3}\right)$, whereas the lasing at $2 \mathrm{nd}$ threshold carrier density was close to the density of 2.1 $\times 10^{25} \mathrm{~m}^{-3}$, where the gain occurs in bulk $\mathrm{ZnO}$. Therefore, it could be considered that the lasing mechanism of the 1st and 2nd lasing would change with increasing the excitation intensity, in which the lasing at 1st threshold was related to the exciton recombination and the lasing at 2nd threshold was induced by the population inversion accompanied by EHP recombination. Because the observed peculiar features such as double threshold, blue-shift, and width change of single lasing peaks were surprisingly similar to the features of polariton or exciton lasers in well-designed semiconductor microcavity lasers, ${ }^{23,24}$ we may expect the transition of the lasing mechanism from a polariton/exciton laser to a photon laser $^{24}$ in the resonance-controlled random structure. Thus, we believe that our results will provide important tools for revealing physical phenomena behind the randomness and the present study may open the possibility that unique phenomena having observed in well-designed microcavities would also be demonstrated even in our random structures, still keeping its merit of easy and low-cost fabrications.

\section{ACKNOWLEDGMENTS}

This work was supported by JSPS KAKENHI (Grant Nos. 15J01516, 16H06506, 26246028, 16K17506, and 26600037), the Cooperative Research Program of "Network Joint Research Center for Materials and Devices," the Murata Science Foundation, the Amada Foundation, and Izumi Science and Technology Foundation.

${ }^{1}$ H. Cao, Y. G. Zhao, S. T. Ho, E. W. Seelig, Q. H. Wang, and R. P. H. Chang, "Random laser action in semiconductor powder," Phys. Rev. Lett. 82, 2278-2281 (1999).

2 J. Fallert, R. J. B. Dietz, J. Sartor, D. Schneider, C. Klingshirn, and H. Kalt, "Co-existence of strongly and weakly localized random laser modes," Nat. Photonics 3, 279-282 (2009).

${ }^{3}$ C. Y. Liu, H. Y. Hu, Y. Sun, J. G. Ma, and Y. C. Liu, “ZnO ultraviolet random laser diode on metal copper substrate,” Opt. Express 14, 16731-16737 (2014).

${ }^{4}$ K. Firdaus, T. Nakamura, and S. Adachi, "Improved lasing characteristics of ZnO/organic-dye random laser," Appl. Phys. Lett. 100, 171101 (2012).

${ }^{5}$ H. Cao, J. Y. Xu, D. Z. Zhang, S.-H. Chang, S. T. Ho, E. W. Seelig, X. Liu, and R. P. H. Chang, "Spatial confinement of laser light in active random media," Phys. Rev. Lett. 84, 5584-5587 (2000).

${ }^{6}$ H. Fujiwara and K. Sasaki, "Observation of optical bistability in a ZnO powder random medium,” Appl. Phys. Lett. 89, 071115 (2006).

${ }^{7}$ M. Sakai, Y. Inose, K. Ema, T. Ohtsuki, H. Sekiguchi, A. Kikuchi, and K. Kishino, "Random laser action in GaN nanocolumns," Appl. Phys. Lett. 97, 151109 (2010).

${ }^{8}$ H. Cao, J. Y. Xu, E. W. Seeling, and R. P. H. Chang, "Microlaser made of disordered media," Appl. Phys. Lett. 76, 2997-2999 (2000).

${ }^{9}$ H. Cao, J. Y. Xu, Y. Ling, A. L. Burin, E. W. Seeling, X. Liu, and R. P. H. Chang, "Random lasers with coherent feedback," IEEE J. Sel. Top. Quantum Electron. 9, 111 (2003).

${ }^{10}$ T. Nakamura, B. P. Tiwari, and S. Adachi, "Control of random lasing in $\mathrm{ZnO} / \mathrm{Al}_{2} \mathrm{O}_{3}$ nanopowders," Appl. Phys. Lett. 99, 231105 (2011).

11 T. Nakamura, S. Sonoda, T. Yamamoto, and S. Adachi, "Discrete-mode ZnO microparticle random laser,” Opt. Lett. 40, 2661 (2015).

12 J. Dai, C. Xu, T. Nakamura, Y. Wang, J. Li, and Y. Lin, “ Electron-hole plasma induced band gap renormalization in ZnO microlaser cavities," Opt. Express 22, 28831 (2014).

${ }^{13}$ T. Nakamura, K. Firdaus, and S. Adach, "Electron-hole plasma lasing in a ZnO random laser," Phys. Rev. B 86, 205103 (2012).

${ }^{14}$ M. A. M. Versteegh, D. Vanmaekelbergh, and J. I. Dijkhuis, "Room-temperature laser emission of ZnO nanowires explained by many-body theory," Phys. Rev. Lett. 108, 157402 (2012).

15 J. C. Johnson, H. Yan, P. Yang, and R. J. Saykally, "Optical cavity effects in ZnO nanowire lasers and waveguides," J. Phys. Chem. B 107, 8816 (2003).

${ }^{16}$ J. Li, M. Jiang, C. Xu, Y. Wang, Y. Lin, J. Lu, and Z. Shi, “Plasmon coupled Fabry-Perot lasing enhancement in graphene/ZnO hybrid microcavity," Sci. Rep. 5, 9263 (2015).

${ }^{17}$ S. Chu, G. Wang, W. Zhou, Y. Lin, L. Chernyak, J. Zhao, J. Kong, L. Li, J. Ren, and J. Liu, "Electrically pumped waveguide lasing from ZnO nanowires," Nat. Nanotechnol. 6, 506-510 (2011).

${ }^{18}$ C. Czekalla, C. Sturm, R. Schemidt-Grund, B. Cao, M. Lorenz, and M. Grundmann, "Whispering gallery mode lasing in zinc oxide microwires," Appl. Phys. Lett. 92, 241102 (2008).

${ }^{19}$ J. Dai, C. X. Xu, R. Ding, K. Zheng, Z. L. Shi, C. G. Lv, and Y. P. Cui, "Combined whispering gallery mode laser from hexagonal ZnO microcavities," Appl. Phys. Lett. 95, 191117 (2009).

${ }^{20}$ H. M. Dong, Y. H. Yang, and G. W. Yang, "Super low threshold plasmonic WGM lasing from an individual ZnO hexagonal microrod on an Au substrate for plasmon lasers," Sci. Rep. 5, 8776 (2015). 
${ }^{21}$ Q. Duan, D. Hu, W. Liu, J. Lu, L. Zhang, J. Wang, Y. Wang, J. Gu, T. Hu, W. Xie, X. Shen, and Z. Chen, "Polariton lasing of quasi-whispering gallery modes in a ZnO microwire,” Appl. Phys. Lett. 103, 022103 (2013).

${ }^{22}$ J. Dai, C. X. Xu, P. Wu, J. Y. Guo, Z. H. Li, and Z. L. Shi, "Exciton and electron-hole plasma lasing in ZnO dodecagonal whispering-gallery-mode microcavities at room temperature,” Appl. Phys. Lett. 97, 011101 (2010).

${ }^{23}$ Y. Y. Lai, Y. H. Chou, Y. P. Lan, T. C. Lu, S. C. Wang, and Y. Yamamoto, "Crossover from polariton lasing to exciton lasing in a strongly coupled ZnO microcavity," Sci. Rep. 6, 20581 (2016).

${ }^{24}$ D. Bajoni, P. Senellart, E. Wertz, I. Sanges, A. Miard, A. Lemaitre, and J. Bloch, "Polariton laser using single micropillar GaAs-GaAlAs semiconductor cavities,” Phys. Rev. Lett. 100, 047401 (2008).

${ }^{25}$ H. Fujiwara, R. Niyuki, Y. Ishikawa, N. Koshizaki, T. Tsuji, and K. Sasaki, "Low-threshold and quasi-single-mode random laser within a submicrometer-sized ZnO spherical particle film,” Appl. Phys. Lett. 102, 061110 (2013).

${ }^{26}$ T. Nakamura, H. Fujiwara, R. Niyuki, K. Sasaki, Y. Ishikawa, N. Koshizaki, T. Tsuji, and S. Adachi, "Origins of lasing emission in resonance-controlled ZnO random laser," New J. Phys. 16, 093054 (2014).

${ }^{27}$ R. Niyuki, H. Fujiwara, Y. Ishikawa, N. Koshizaki, T. Tsuji, and K. Sasaki, "Toward single-mode random lasing within a submicrometre-sized spherical ZnO particle film,” J. Opt. 18, 035202 (2016).

${ }^{28}$ T. Tsuji, Y. Higashi, M. Tsuji, H. Fujiwara, Y. Ishikawa, and N. Koshizaki, "Fabrication of spherical-shaped submicron particles of Zno using laser-induced melting of submicron-sized source materials," J. Laser Micro/Nanoeng. 8, 292-295 (2013).

${ }^{29}$ H. Fujiwara, Y. Hamabata, and K. Sasaki, "Numerical analysis of resonant and lasing properties at a defect region within a random structure," Opt. Express 17, 3970-3977 (2009).

${ }^{30}$ M. A. M. Versteegh, T. Kuis, C. T. H. Stoof, and I. J. Dijkhuis, "Ultrafast screening and carrier dynamics in ZnO: Theory and experiment," Phys. Rev. B 84, 035207 (2011).

${ }^{31}$ H. Priller, R. Hauschild, J. Zeller, C. Klingshirn, H. Kalt, R. Kling, F. Reuss, C. Kirchner, and A. Waag, "Temperaturedependent luminescence dynamics in $\mathrm{ZnO}$ nanorods," J. Lumin. 112, 173-176 (2005).

${ }^{32}$ R. Chen, Q.-L. Ye, T. He, V. D. Ta, Y. Ying, Y. Y. Tay, T. Wu, and H. Sun, "Exciton localization and optical properties improvement in nanocrystal-embedded $\mathrm{ZnO}$ core-shell nanowires," Nano Lett. 13, 734 (2013).

${ }^{33}$ J. Fallert, F. Stelzl, H. Zhou, A. Reiser, K. Thonke, R. Sauer, C. Klingshirn, and H. Kalt, "Lasing dynamics in single ZnO nanorods," Opt. Express 16, 1125-1131 (2008).

${ }^{34}$ G. Bjork, A. Karlsson, and Y. Yamamoto, "On the linewidth of microcavity lasers,” Appl. Phys. Lett. 60, 304 (1992).

${ }^{35}$ J. Kasprzak, M. Richard, A. Bass, B. Deveaud, R. Andre, J.-Ph. Poizat, and Le S. Dang, "Second-order time correlations within a polariton Bose-Einstein condensate in a CdTe microcavity," Phys. Rev. Lett. 100, 067402 (2008).

${ }^{36}$ D. Porras and C. Tejedor, "Linewidth of a polariton laser: Theoretical analysis of self-interaction effects," Phys. Rev. B 67, 161310 (2003)

${ }^{37}$ M. D. Bagnall, F. Y. Chen, T. Yao, Y. M. Shen, and T. Goto, "High temperature excitonic stimulated emission from ZnO epitaxial layers," Appl. Phys. Lett. 73, 1038-1040 (1998).

${ }^{38}$ C. Klingshirn, R. Hauschild, J. Fallert, and H. Kalt, "Room-temperature stimulated emission of ZnO: Alternatives to excitonic lasing," Phys. Rev. B 75, 115203 (2007). 